\title{
Bisimulation for Demonic Schedulers ${ }^{\star}$
}

\author{
Konstantinos Chatzikokolakis ${ }^{1}$, Gethin Norman ${ }^{2}$, and David Parker ${ }^{2}$ \\ 1 Eindhoven University of Technology \\ ${ }^{2}$ Oxford Computing Laboratory
}

\begin{abstract}
Bisimulation between processes has been proven a successful method for formalizing security properties. We argue that in certain cases, a scheduler that has full information on the process and collaborates with the attacker can allow him to distinguish two processes even though they are bisimilar. This phenomenon is related to the issue that bisimilarity is not preserved by refinement. As a solution, we introduce a finer variant of bisimulation in which processes are required to simulate each other under the "same" scheduler. We formalize this notion in a variant of CCS with explicit schedulers and show that this new bisimilarity can be characterized by a refinement-preserving traditional bisimilarity. Using a third characterization of this equivalence, we show how to verify it for finite systems. We then apply the new equivalence to anonymity and show that it implies strong probabilistic anonymity, while the traditional bisimulation does not. Finally, to illustrate the usefulness of our approach, we perform a compositional analysis of the Dining Cryptographers with a non-deterministic order of announcements and for an arbitrary number of cryptographers.
\end{abstract}

\section{Introduction}

Process algebra provides natural models for security protocols in which nondeterminism plays an essential role, allowing implementation details to be abstracted ([123]). In this setting, security properties are often stated in using equivalence relations, with bisimulation commonly used. Its application takes two distinct forms. In the first, the protocol is shown to be bisimilar to a specification which satisfies the required security property, then since the protocol and specification are equivalent, we conclude that the protocol satisfies the property. An example is the formalization of authenticity in [2], in which $A$ sends a message $m$ and $B$ wants to ensure that it receives $m$ and not a different message from some other agent. In the specification, we allow $B$ to test the received message against the real $m$ (as if $B$ knew it beforehand). Showing the protocol is bisimilar to the specification ensures $B$ receives the correct message.

The second form is substantially different: we establish a bisimulation relation between two distinct instances of the protocol. From this, we conclude that the instances are indistinguishable to the attacker, that is the difference between the two instances remains hidden from the attacker. An example of this approach is the formalization of

\footnotetext{
* This work was carried out while Konstantinos Chatzikokolakis was visiting Oxford University. Chatzikokolakis wishes to thank Marta Kwiatkowska for giving him the opportunity to collaborate with her group. Authors Norman and Parker where supported in part by EPSRC grants EP/D077273/1 and EP/D07956X/2.
}

L. de Alfaro (Ed.): FOSSACS 2009, LNCS 5504, pp. 318-332. 2009.

(C) Springer-Verlag Berlin Heidelberg 2009 
secrecy in [2]. If $P(m)$ is a protocol parametrized by a message $m$, and we demonstrate that $P(m)$ and $P\left(m^{\prime}\right)$ are bisimilar, then the message remains secret. Another example is privacy in voting protocols ([4]). The votes of $A$ and $B$ remain private if an instance of the protocol is bisimilar to the instance in which $A$ and $B$ have exchanged votes.

In this paper we focus on the second use of bisimulation and we argue that, in the presence of a scheduler who has full view of the process, an attacker could actually distinguish bisimilar processes. The reason is that, in the definition of bisimulation, non-determinism is treated in a partially angelic way. Letting $\sim$ denote bisimilarity, when $P \sim Q$ one requires that if $P$ can make a transition $\alpha$ to $P^{\prime}$, then $Q$ can make a transition $\alpha$ to some $Q^{\prime}$ such that $Q \sim Q^{\prime}$ (and vice-versa). In this definition, there are two implicit quantifiers, the second being existential:

$$
\begin{aligned}
\text { for all transitions } & P \stackrel{\alpha}{\longrightarrow} P^{\prime} \\
\text { there exists a transition } & Q \stackrel{\alpha}{\longrightarrow} Q^{\prime} \quad \text { s.t. } P^{\prime} \sim Q^{\prime}
\end{aligned}
$$

In other words, $Q$ is not forced to simulate $P$, it only has to have the possibility to do so. For $P$ and $Q$ to remain indistinguishable in the actual execution, we have to count on the fact that the scheduler of $Q$ will guide it in a way that simulates $P$, that is the scheduler acts in favour of the process. In security, however, we want to consider the worst case scenario, thus we typically assume that the scheduler collaborates with the attacker. A "demonic" scheduler in the implementation of $Q$ can choose to do something different, allowing the attacker to distinguish the two processes.

Consider the simple example of an agent broadcasting a message $m$ on a network, received by agents $A$ and $B$ and then acknowledged (with each agent including its identity in the acknowledgement). We can model this protocol as:

$$
P(m)=(\nu c)(\bar{c}\langle m\rangle \cdot \bar{c}\langle m\rangle|A| B) \quad \text { where } A=c(x) . a \text { and } B=c(x) . b
$$

Clearly, $P(m) \sim P\left(m^{\prime}\right)$, but does $m$ remain secret? Both instances can perform two visible actions, $a$ and $b$, the order of which is chosen non-deterministically. The indistinguishability of the two processes relies on the schedulers of $P(m)$ and $P\left(m^{\prime}\right)$ choosing the same order for the visible actions. If, however, one scheduler chooses a different order, then we can distinguish $m$ from $m^{\prime}$ through on the output of the protocol. This could be the case, for example, if an operation is performed upon reception whose execution time is message dependent.

This consequence of angelic non-determinism can be also formulated in terms of refinement, where $Q$ refines $P$ if it contains "less" non-determinism. While an implementation of a protocol is a refinement of it, bisimulation need not be preserved by refinement, thus security properties might no longer hold in the implementation, an issue often called the "refinement paradox" ([56]). In the example above, $P(m)$ and $P\left(m^{\prime}\right)$ could be implemented by $(\nu c)(\bar{c}\langle m\rangle \cdot \bar{c}\langle m\rangle \mid c(x) \cdot a \cdot c(x) \cdot b)$ and $(\nu c)\left(\bar{c}\left\langle m^{\prime}\right\rangle \cdot \bar{c}\left\langle m^{\prime}\right\rangle \mid\right.$ $c(x) . b . c(x) . a)$ respectively which can be distinguished. Note that, in the specificationbased use of bisimulation this issue does not appear: as the protocol and specification are bisimilar, the implementation will be a refinement of the specification which is usually enough to guarantee the required security property.

It should be noted that classical bisimulation does offer some control over nondeterminism as it is closed under contexts and contexts can restrict available actions. 
More precisely, bisimulation is robust against schedulers that can be expressed through contexts. However, contexts cannot control internal choices, like the selection of the first receiver in the above example. The example could be rewritten to make this selection external, however, for more complex protocols this solution becomes challenging. Also, when using contexts, it is not possible to give some information to the scheduler, without making the corresponding action visible, that is, without revealing it to an observer. This could be problematic when, for example, verifying a protocol in which the scheduler, even if he knows some secret information, has no possibility to communicate it to the outside.

In this paper we propose an approach based on a variant of bisimulation, called demonic bisimulation, in which non-determinism is treated in a purely demonic way. In principle, we would like to turn the existential quantifier into a universal one, but this is too restrictive and the relation would not even be reflexive. Instead we require $Q$ to simulate a transition $\alpha$ of $P$, not under any scheduler but under the same scheduler that produced $\alpha$ :

\section{for all schedulers $S$, if $P \stackrel{\alpha}{\longrightarrow} P^{\prime}$ \\ then under the same scheduler $S: \quad Q \stackrel{\alpha}{\longrightarrow} Q^{\prime} \quad$ with $P^{\prime} \sim Q^{\prime}$}

Note that, in general, it is not possible to speak about the "same" scheduler, since different processes have different choices. Still, this is reasonable if the processes have a similar structure (as in the case of $P(m)$ and $P\left(m^{\prime}\right)$ ), in this paper we give a framework that allows us to formalize this concept. The basic idea is that we can choose the scheduler that can break our property, however we must test both processes under the same one. This requirement is both realistic, as we are interested in the indistinguishability of two processes when put in the same environment, and strong, since it leaves no angelic non-determinism.

To formalize demonic bisimulation we use a variant of probabilistic CCS with explicit schedulers, which was introduced in [7] to study the information that a scheduler has about the state of a process. This calculus allows us to speak of schedulers independently from processes, leading to a natural definition of demonic bisimulation. Then, we discuss how we can view a scheduler as a refinement operator that restricts the nondeterminism of a process. We define a refinement operator, based on schedulers, and we show that demonic bisimilarity can be characterized as a refinement-preserving classical bisimilarity, for this type of refinement. Afterwards, we give a third characterization of demonic bisimilarity, that allows us to obtain an algorithm to verify finite processes. Finally, we apply the demonic bisimulation to the analysis of anonymity protocols and show that demonic bisimulation, in contrast to the classical one, implies strong probabilistic anonymity. This enables us to perform a compositional analysis of the Dining Cryptographers protocol demonstrating that it satisfies anonymity for an arbitrary number of cryptographers.

\section{Preliminaries}

We denote by $\operatorname{Disc}(X)$ the set of all discrete probability measures over $X$, and by $\delta(x)$ (called the Dirac measure on $x$ ) the probability measure that assigns probability 1 to 
$\{x\}$. We will also denote by $\sum_{i}\left[p_{i}\right] \mu_{i}$ the probability measure obtained as a convex sum of the measures $\mu_{i}$.

A simple probabilistic automaton is a tuple $(S, q, A, \mathcal{D})$ where $S$ is a set of states, $q \in S$ is the initial state, $A$ is a set of actions and $\mathcal{D} \subseteq S \times A \times \operatorname{Disc}(S)$ is a transition relation. Intuitively, if $(s, a, \mu) \in \mathcal{D}$, also written $s \stackrel{a}{\longrightarrow} \mu$, then there is a transition from the state $s$ performing the action $a$ and leading to a distribution $\mu$ over the states of the automaton. A probabilistic automaton $M$ is fully probabilistic if from each state of $M$ there is at most one transition available. An execution $\alpha$ of a probabilistic automaton is a (possibly infinite) sequence $s_{0} a_{1} s_{1} a_{2} s_{2} \ldots$ of alternating states and actions, such that $q=s_{0}$, and for each $i: s_{i} \stackrel{a_{i+1}}{\longrightarrow} \mu_{i}$ and $\mu_{i}\left(s_{i+1}\right)>0$. A scheduler of a probabilistic automaton $M=(S, q, A, \mathcal{D})$ is a function $\zeta: \operatorname{exec}^{*}(M) \mapsto \mathcal{D}$ where $\operatorname{exec}^{*}(M)$ is the set of finite executions of $M$, such that $\zeta(\alpha)=(s, a, \mu) \in \mathcal{D}$ implies that $s$ is the last state of $\alpha$. The idea is that a scheduler selects a transition among the ones available in $\mathcal{D}$ and it can base its decision on the history of the execution. A scheduler induces a probability space on the set of executions of $M$.

If $\mathcal{R}$ is a relation over a set $S$, then we can lift the relation to probability distributions over $S$ using the standard weighting function technique (see [8] for details). If $\mathcal{R}$ is an equivalence relation then the lifting can be simplified: $\mu_{1} \mathcal{R} \mu_{2}$ iff for all equivalence classes $\mathcal{E} \in S / \mathcal{R}, \mu_{1}(\mathcal{E})=\mu_{2}(\mathcal{E})$. We can now define simulation and bisimulation for simple probabilistic automata.

Definition 1. Let $(S, q, A, \mathcal{D})$ be a probabilistic automaton. A relation $\mathcal{R} \subseteq S \times S$ is a simulation iff for all $\left(s_{1}, s_{2}\right) \in \mathcal{R}, a \in A$ : if $s_{1} \stackrel{a}{\longrightarrow} \mu_{1}$ then there exists $\mu_{2}$ such that $s_{2} \stackrel{a}{\longrightarrow} \mu_{2}$ and $\mu_{1} \mathcal{R} \mu_{2}$. A simulation $\mathcal{R}$ is a bisimulation if it is also symmetric (thus, it is an equivalence relation). We define $\sqsubseteq, \sim$ as the largest simulation and bisimulation on $S$ respectively.

CCS with Internal Probabilistic Choice. Let $a$ range over a countable set of channel names and let $\alpha$ stand for $a, \bar{a}$ or $\tau$. The syntax of $\mathrm{CCS}_{p}$ is:

$$
P, Q:=a . P|P| Q|P+Q| \sum_{i} p_{i} P_{i}|(\nu a) P| ! a . P \mid 0
$$

The term $\sum_{i} p_{i} P_{i}$ represents an internal probabilistic choice, all the remaining operators are from standard CCS. We will also use the notation $P_{1}{ }_{{ }_{p}} P_{2}$ to represent a binary sum $\sum_{i} p_{i} P_{i}$ with $p_{1}=p$ and $p_{2}=1-p$. Finally, we use replicated input instead of replication or recursion, as this simplifies the presentation. The semantics of $\mathrm{CCS}_{p}$ is standard and has been omitted due to space constraints. The full semantics can be found in the report version of this paper ([9]). We denote this transition system by $\longrightarrow_{c}$ to distinguish it from other transition systems defined later in the paper.

\section{A Variant of $\mathrm{CCS}_{p}$ with Explicit Scheduler}

In this section we present a variant of $\mathrm{CCS}_{p}$ in which the scheduler is explicit, in the sense that it has a specific syntax and its behaviour is defined by the operational semantics of the calculus. This calculus was proposed in [7]; we will refer to it as $\mathrm{CCS}_{\sigma}$. Processes in $\mathrm{CCS}_{\sigma}$ contain labels that allow us to refer to a particular sub-process. A 


\begin{tabular}{|c|c|c|c|}
\hline$I::=0 I|1 I| \epsilon$ & label indexes & $S, T::=$ & scheduler \\
\hline$L::=l^{I}$ & labels & $\begin{array}{ll} & L . S \\
\mid & (L, L) . S\end{array}$ & $\begin{array}{l}\text { schedule single action } \\
\text { synchronization }\end{array}$ \\
\hline$P, Q::=$ & processes & if $L$ & label test \\
\hline$L: \alpha . P$ & prefix & then $S$ & \\
\hline$P \mid Q$ & parallel & else $S$ & \\
\hline$P+Q$ & nondeterm. choice & 0 & nil \\
\hline $\begin{array}{l}L: \sum_{i} p_{i} P_{i} \\
(\nu a) P\end{array}$ & $\begin{array}{l}\text { internal prob. choice } \\
\text { restriction }\end{array}$ & $C P::=P \| S$ & complete process \\
\hline$! L: a . P$ & replicated input & & \\
\hline$L: 0$ & nil & & \\
\hline
\end{tabular}

Fig. 1. The syntax of $\mathrm{CCS}_{\sigma}$

scheduler also behaves like a process, using however a different and much simpler syntax, and its purpose is to guide the execution of the main process using the labels that the latter provides.

\subsection{Syntax}

Let $a$ range over a countable set of channel names and $l$ over a countable set of atomic labels. The syntax of $\mathrm{CCS}_{\sigma}$, shown in Figure 1 is the same as the one of $\mathrm{CCS}_{p}$ except for the presence of labels. These are used to select the subprocess which "performs" a transition. Since only the operators with an initial rule can originate a transition, we only need to assign labels to the prefix and to the probabilistic sum. We use labels of the form $l^{s}$ where $l$ is an atomic label and the index $s$ is a finite string of 0 and 1 , possibly empty. Indexes are used to avoid multiple copies of the same label in case of replication. As explained in the semantics, each time a process is replicated we relabel it using appropriate indexes. To simplify the notation, we use base labels of the form $l_{1}, \ldots, l_{n}$, and we write ${ }^{i} a . P$ for $l_{i}: a . P$.

A scheduler selects a sub-process for execution on the basis of its label, so we use $l . S$ to represent a scheduler that selects the process with label $l$ and continues as $S$. In the case of synchronization we need to select two processes simultaneously, hence we need a scheduler of the form $\left(l_{1}, l_{2}\right) . S$. We will use $S_{l}$ to denote a scheduler of one of these forms (that is, a scheduler that starts with a label or pair of labels). The if-thenelse construct allows the scheduler to test whether a label is available in the process (in the top-level) and act accordingly. A complete process is a process put in parallel with a scheduler, for example $l_{1}: a . l_{2}: b \| l_{1} . l_{2}$. We define $\mathcal{P}, \mathcal{C P}$ to be the sets of all processes and all complete $\mathrm{CCS}_{\sigma}$ processes respectively. Note that for processes with an infinite execution path we need schedulers of infinite length.

\subsection{Semantics for Complete Processes}

The semantics of $\mathrm{CCS}_{\sigma}$ is given in terms of a probabilistic automaton whose state space is $\mathcal{C P}$ and whose transitions are given by the rules in Figure 2 We denote the transitions by $\longrightarrow_{s}$ to distinguish it from other transition systems.

ACT is the basic communication rule. In order for $l: \alpha . P$ to perform $\alpha$, the scheduler should select this process for execution, so the scheduler needs to be of the form $l . S$. 


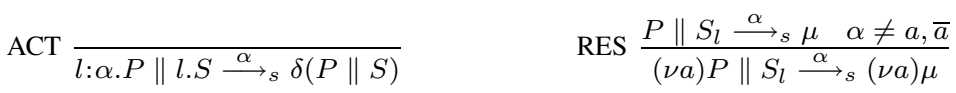

$$
\begin{aligned}
& \text { SUM1 } \frac{P \| S_{l} \stackrel{\alpha}{\longrightarrow}_{s} \mu}{P+Q \| S_{l} \stackrel{\alpha}{\longrightarrow}_{s} \mu} \quad \quad \text { PAR } 1 \frac{P \| S_{l} \stackrel{\alpha}{\longrightarrow}_{s} \mu}{P\left|Q \| S_{l} \stackrel{\alpha}{\longrightarrow}_{s} \mu\right| Q} \\
& \operatorname{COM} \frac{P\left\|l_{1} \stackrel{a}{\longrightarrow} \delta\left(P^{\prime} \| 0\right) \quad Q\right\| l_{2} \stackrel{\bar{a}}{\longrightarrow} s\left(Q^{\prime} \| 0\right)}{P \mid Q \|\left(l_{1}, l_{2}\right) . S \stackrel{\tau}{\longrightarrow} \delta\left(P^{\prime} \mid Q^{\prime} \| S\right)} \\
& \text { PROB } \overline{l: \sum_{i} p_{i} P_{i} \| l . S \stackrel{\tau}{\longrightarrow} s \sum_{i} p_{i} \delta\left(P_{i} \| S\right)} \\
& \text { IF1 } \frac{l \in t l(P) \quad P \| S_{1} \stackrel{\alpha}{\longrightarrow}_{s} \mu}{P \| \text { if } l \text { then } S_{1} \text { else } S_{2} \stackrel{\alpha}{\longrightarrow}_{s} \mu} \quad \quad \text { IF2 } \frac{l \notin t l(P) \quad P \| S_{2} \stackrel{\alpha}{\longrightarrow} s \mu}{P \| \text { if } l \text { then } S_{1} \text { else } S_{2} \stackrel{\alpha}{\longrightarrow}_{s} \mu} \\
& \text { REP } \longdiv { ! l : a . P \| l . S \stackrel { \alpha } { \longrightarrow } \delta ( \rho _ { 0 } P | ! l : a . \rho _ { 1 } P \| S ) }
\end{aligned}
$$

Fig. 2. The semantics of complete $\mathrm{CCS}_{\sigma}$ processes. SUM1 and PAR1 have corresponding right rules SUM2 and PAR2, omitted for simplicity.

After this execution the complete process will continue as $P \| S$. The RES rule models restriction on channel $a$ : communication on this channel is not allowed by the restricted process. We denote by $(\nu a) \mu$ the measure $\mu^{\prime}$ such that $\mu^{\prime}((\nu a) P \| S)=\mu(P \| S)$ for all processes $P$ and $\mu^{\prime}(R \| S)=0$ if $R$ is not of the form $(\nu a) P$. SUM1 models nondeterministic choice. If $P \| S$ can perform a transition to $\mu$, which means that $S$ selects one of the labels of $P$, then $P+Q \| S$ will perform the same transition, i.e. the branch $P$ of the choice will be selected and $Q$ will be discarded. For example $l_{1}: a . P+l_{2}: b . Q \| l_{1} . S \stackrel{a}{\longrightarrow} \delta(P \| S)$. Note that the operands of the sum do not have labels, the labels belong to the subprocesses of $P$ and $Q$. In the case of nested choices, the scheduler must select the label of a prefix, thus resolving all the choices at once.

PAR1, modelling parallel composition, is similar: the scheduler selects $P$ to perform a transition on the basis of the label. The difference is that in this case $Q$ is not discarded; it remains in the continuation. $\mu \mid Q$ denotes the measure $\mu^{\prime}$ such that $\mu^{\prime}(P \mid Q \| S)=$ $\mu(P \| S)$. COM models synchronization. If $P \| l_{1}$ can perform the action $a$ and $Q \| l_{2}$ can perform $\bar{a}$, then $\left(l_{1}, l_{2}\right) . S$ can synchronize the two by scheduling both $l_{1}$ and $l_{2}$ at the same time. PROB models internal probabilistic choice. Note that the scheduler cannot affect the outcome of the choice, it can only schedule the choice as a whole (this is why a probabilistic sum has a label) and the process will move to a measure containing all the operands with corresponding probabilities.

REP models replicated input. This rule is the same as in CCS, with the addition of a re-labeling operator $\rho_{i}$. The reason for this is that we want to avoid ending up with multiple copies of the same label as the result of replication, since this would create ambiguities in scheduling as explained in Section 3.3 $\rho_{i} P$ appends $i \in\{0,1\}$ to the index of all labels of $P$, for example: $\rho_{i} l^{s}: \alpha . P=l^{s i}: \alpha . \rho_{i} P$ and similarly for the other operators. Note that we relabel only the resulting process, not the continuation of the scheduler: there is no need for relabeling the scheduler since we are free to choose the continuation as we please.

Finally if-then-else allows the scheduler to adjust its behaviour based on the labels that are available in $P . t l(P)$ gives the set of top-level labels of $P$ and is defined as: $t l(l: \alpha . P)=t l\left(l: \sum_{i} p_{i} P_{i}\right)=t l(! l: a . P)=t l(l: 0)=\{l\}$ and as the union of the 
top-level labels of all sub-processes for the other operators. Then if $l$ then $S_{1}$ else $S_{2}$ behaves like $S_{1}$ if $l$ is available in $P$ and as $S_{2}$ otherwise.

A process is blocked if it cannot perform a transition under any scheduler. A scheduler $S$ is non-blocking for a process $P$ if it always schedules some transition, except when $P$ itself is blocked.

\subsection{Deterministic Labelings}

The idea in $\mathrm{CCS}_{\sigma}$ is that a syntactic scheduler will be able to completely resolve the nondeterminism of the process, without needing to rely on a semantic scheduler at the level of the automaton. To achieve this we impose a condition on the labels of $\mathrm{CCS}_{\sigma}$ processes. A labeling for $P$ is an assignment of labels to the subprocesses of $P$ that require a label. A labeling for $P$ is deterministic iff for all schedulers $S$ there is at most one transition of $P \| S$ enabled at any time, in other words the corresponding automaton is fully probabilistic. In the rest of the paper, we only consider processes with deterministic labelings.

A simple case of deterministic labelings are the linear ones, containing pairwise distinct labels (a more precise definition of linear labelings requires an extra condition and can be found in [10]). It can be shown that linear labelings are preserved by transitions and are deterministic. However, the interesting case is that we can construct labelings that are deterministic without being linear. The usefulness of non-linear labelings is that they limit the power of the scheduler, since the labels provide information about the current state and allow the scheduler to choose different strategies through the use of if-then-else. Consider, for example, the following process whose labeling is deterministic but not linear:

$$
l:\left({ }^{1} \bar{a} \cdot R_{1}+{ }_{p}{ }^{1} \bar{a} \cdot R_{2}\right)\left|{ }^{2} a \cdot P\right|{ }^{3} a \cdot Q
$$

Since both branches of the probabilistic sum have the same label $l_{1}$, the scheduler cannot resolve the choice between $P$ and $Q$ based on the outcome of the probabilistic choice. Another use of non-linear labeling is the encoding of "private" value passing ([7]):

$$
l: c(x) . P \triangleq \sum_{i} l: c v_{i} \cdot P\left[v_{i} / x\right] \quad l: \bar{c}\langle v\rangle \cdot P \triangleq l: \overline{c v} \cdot P
$$

This is the usual encoding of value passing in CCS except that we use the same label in all the branches of the nondeterministic sum. Thus, the reception of a message is visible to the scheduler, but not the received value.

\section{Demonic Bisimulation}

As discussed in the introduction, classical bisimulation treats non-determinism in a partially angelic way. In this section we define a strict variant of bisimulation, called demonic bisimulation, which treats non-determinism in a purely demonic way. We characterize this equivalence in two ways, first in terms of schedulers, then in terms of refinement. 


\subsection{Definition Using Schedulers}

An informal definition of demonic bisimulation was already given in the introduction: $P$ is demonic-bisimilar to $Q$, written $P \sim_{D} Q$ if for all schedulers $S$, if $P \stackrel{\alpha}{\longrightarrow} P^{\prime}$ then under the same scheduler $S: Q \stackrel{\alpha}{\longrightarrow} Q^{\prime}$ with $P^{\prime} \sim_{D} Q$. To define demonic bisimulation concretely, we need a framework that allows a single scheduler to be used with different processes. $\mathrm{CCS}_{\sigma}$ does exactly this: it gives semantics to $P \| S$ for any process $P$ and scheduler $S$ (of course, $S$ might be blocking for some processes and non-blocking for others).

If $\mu$ is a discrete measure on $\mathcal{P}$, we denote by $\mu \| S$ the discrete measure $\mu^{\prime}$ on $\mathcal{C P}$ such that $\mu^{\prime}(P \| S)=\mu(P)$ for all $P \in \mathcal{P}$ and $\mu^{\prime}\left(P \| S^{\prime}\right)=0$ for all $S^{\prime} \neq S$ (note that all transition rules of Fig. 2. produce measures of this form).

Definition 2 (Demonic bisimulation). An equivalence relation $\mathcal{R}$ on $\mathcal{P}$ is a demonic bisimulation iff for all $\left(P_{1}, P_{2}\right) \in \mathcal{R}, a \in A$ and all schedulers $S$ : if $S$ is non-blocking for $P_{1}$ and $P_{1}\left\|S \stackrel{\alpha}{\longrightarrow} \mu_{1}\right\| S^{\prime}$ then the same scheduler $S$ is non-blocking for $P_{2}$ and $P_{2}\left\|S \stackrel{\alpha}{\longrightarrow} \mu_{2}\right\| S^{\prime}$ with $\mu_{1} \mathcal{R} \mu_{2}$. We define demonic bisimlarity $\sim_{D}$ as the largest demonic bisimulation on $\mathcal{P}$.

Consider again the example of the introduction. We define:

$$
A={ }^{1} c(x) .{ }^{2} a \quad B={ }^{3} c(x) .{ }^{4} b \quad P(m)=(\nu c)\left({ }^{5} \bar{c}\langle m\rangle .{ }^{6} \bar{c}\langle m\rangle|A| B\right)
$$

Note that $P(m), P\left(m^{\prime}\right)$ share the same labels. This choice of labels states that whenever a scheduler chooses an action in $P(m)$, it has to schedule the same action in $P\left(m^{\prime}\right)$. Then it is easy to see that $P(m) \sim_{D} P\left(m^{\prime}\right)$. A scheduler that selects $A$ first in $P(m)$ will also select $A$ first in $P\left(m^{\prime}\right)$, leading to the same order of actions. Under this definition, we do not rely on angelic non-determinism for $P\left(m^{\prime}\right)$ to simulate $P(m)$, we have constructed our model in a way that forces a scheduler to perform the same action in both processes. Note that we could also choose to put different labels in $\bar{c}\langle m\rangle, \bar{c}\left\langle m^{\prime}\right\rangle$, hence allowing them to be scheduled in a different way. In this case $\sim_{D}$ will no longer hold, exactly because we can now distinguish the two processes using an if-then-else scheduler that depends on the message. Finally we perform a usual sanity check:

Proposition 1. $\sim_{D}$ is a congruence.

\subsection{Characterization Using Refinement}

Another way of looking at schedulers is in terms of refinement: a process $Q$ refines $P$ if it contains "less" non-determinism. A typical definition is in terms of simulation: $Q$ refines $P$ if $Q \sqsubseteq P$. For example, $a$ is a refinement of $a+b$ where the non-deterministic choice has been resolved. Thus, a scheduler can be seen as a way to refine a process by resolving the non-determinism. For example, $l_{1}: a$ can be seen as the refinement of $l_{1}: a+l_{2}: b$ under the scheduler $l_{1}$. Moreover, partial schedulers can be considered as resolving only part of the non-determinism. For example, for the process ${ }^{1} a \cdot\left({ }^{3} c+{ }^{4} d\right)+$ ${ }^{2} b$, the scheduler $l_{1}$ will resolve the first non-deterministic choice but not the second.

It has been observed that many security properties are not preserved by refinement, a phenomenon often called the "refinement paradox". If we define security properties 


$$
\begin{aligned}
\varphi_{0}(P) & =P \\
\varphi_{\lambda . S}(\lambda: \alpha . P) & =\lambda: \alpha . \varphi_{S}(P) \\
\varphi_{\lambda . S}(P+Q) & =\varphi_{\lambda . S}(P)+\varphi_{\lambda . S}(Q) \\
\varphi_{\lambda . S}((\nu a) P) & =\left(\nu a \varphi_{\lambda . S}(P)\right. \\
\varphi_{l . S}\left(l: \sum_{i} p_{i} P_{i}\right) & =l: \sum_{i} p_{i} \varphi_{S}\left(P_{i}\right) \\
\varphi_{\lambda . S}(P \mid Q) & = \begin{cases}\lambda: \alpha . \varphi_{S}\left(P^{\prime} \mid Q\right) & \text { if } \varphi_{\lambda}(P) \stackrel{\alpha}{\longrightarrow}_{c} \delta\left(P^{\prime}\right) \\
\lambda: \sum_{i} p_{i} \varphi_{S}\left(P_{i} \mid Q\right) & \text { if } \varphi_{\lambda}(P) \stackrel{\tau}{\longrightarrow}_{c} \sum_{i}\left[p_{i}\right] \delta\left(P_{i}\right) \\
\lambda: \tau . \varphi_{S}\left(P^{\prime} \mid Q^{\prime}\right) & \text { if } \lambda=\left(l_{1}, l_{2}\right) \text { and } \\
\varphi_{l . S}(! l: a . P) & =l: a . \varphi_{S}\left(\rho_{0} P \mid ! l: a . \rho_{1} P\right) \\
\varphi_{S}(P) & =\left\{\begin{array}{ll}
\varphi_{S_{1}}(P) & \text { if } l \in t l(P) \text { where } S=\text { if } l \text { then } S_{1} \text { else } S_{2} \\
\varphi_{S_{2}}(P) & \text { if } l \notin t l(P)
\end{array} \stackrel{\bar{a}}{\longrightarrow}_{c} \delta\left(Q^{\prime}\right)\right. \\
\varphi_{S}(P) & \left.=0 \quad \text { if none of the above is applicable (e.g. } \varphi_{l_{1}}\left(l_{2}: \alpha . P\right)=0\right)\end{cases}
\end{aligned}
$$

Fig. 3. Refinement of $\mathrm{CCS}_{\sigma}$ processes under a scheduler. The symmetric cases for the parallel operator have been omitted for simplicity.

using bisimulation this issue immediately arises. For example, $a \mid b$ is bisimilar to $a . b+$ $b . a$ but if we refine them to $a . b$ and $b . a$ respectively, they are no longer bisimilar. Clearly, if we want to preserve bisimilarity we have to refine both processes in a consistent way. In this section, we introduce a refinement operator based on schedulers. We are then interested in processes that are not only bisimilar, but also preserve bisimilarity under this refinement. We show that this stronger equivalence coincides with demonic bisimilarity.

With a slight abuse of notation we extend the transitions $\longrightarrow_{c}$ (the traditional transition system for $\mathrm{CCS}_{p}$ ) to $\mathrm{CCS}_{\sigma}$ processes, by simply ignoring the labels, which are then only used for the refinement. Let $S$ be a finite scheduler and $P$ a $\operatorname{CCS}_{\sigma}$ process. The refinement of $P$ under $S$, denoted by $\varphi_{S}(P)$, is a new $\operatorname{CCS}_{\sigma}$ process. The function $\varphi_{S}: \mathcal{P} \rightarrow \mathcal{P}$ is defined in Figure 3 Note that $\varphi_{S}$ does not perform transitions, it only blocks the transitions that are not enabled by $S$. Thus, it reduces the non-determinism of the process. The scheduler might be partial: a scheduler 0 leaves the process unaffected (2). Thus, the resulting process might still have non-deterministic choices. A prefix is allowed in the refined process only if its label is selected by the scheduler (3), otherwise the refined process is equal to $0(10)$. Case (4) applies the refinement to both operands. Note that, if the labeling is deterministic, at most one of the two will have transitions enabled. The most interesting case is the parallel operator (7). There are three possible executions for $P \mid Q$. An execution of $P$ alone, of $Q$ alone or a synchronization between the two. The refined version enforces the one selected by the scheduler (the symmetric cases have been omitted for simplicity). This is achieved by explicitly prefixing the selected action, for example $l_{1}: a \mid l_{2}: b$ refined by $l_{1}$ becomes $l_{1}: a .\left(0 \mid l_{2}: b\right)$. If $P$ performs a probabilistic choice, then we have to use a probabilistic sum instead of an action prefix. The case of $! P(8)$ is similar to the prefix (3) and the rest of the cases are self-explanatory. 


$$
\begin{aligned}
& \text { ACT } \overline{l: \alpha . P \stackrel{l: \alpha}{\longrightarrow} \delta(P)} \\
& \operatorname{RES} \frac{P \stackrel{l: \alpha}{\longrightarrow}_{a} \mu \quad \alpha \neq a, \bar{a}}{(\nu a) P \stackrel{l: \alpha}{\longrightarrow}_{a}(\nu a) \mu}
\end{aligned}
$$

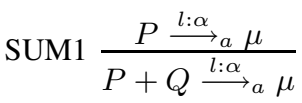

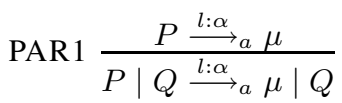

$$
\begin{aligned}
& \operatorname{COM} \frac{P \stackrel{P}{\stackrel{l_{1}: a}{\longrightarrow}} a \delta\left(P^{\prime}\right) Q \stackrel{l_{2}: \bar{a}}{\longrightarrow} a \delta\left(Q^{\prime}\right)}{P \mid Q \stackrel{\left(l_{1}, l_{2}\right): \tau}{\longrightarrow}{ }_{a} \delta\left(P^{\prime} \mid Q^{\prime}\right)} \\
& \text { PROB } \overline{l: \sum_{i} p_{i} P_{i} \stackrel{l: \tau}{\longrightarrow} a \sum_{i} p_{i} \delta\left(P_{i}\right)} \\
& \text { REP } \overline{! l: a . P \stackrel{l: a}{\longrightarrow}{ }_{a} \delta\left(\rho_{0} P \mid ! l: a . \rho_{1} P\right)}
\end{aligned}
$$

Fig. 4. Semantics for $\mathrm{CCS}_{\sigma}$ processes without schedulers. SUM1 and PAR1 have corresponding right rules SUM2 and PAR2, omitted for simplicity.

The intention behind the definition of $\phi_{S}$ is to refine $\mathrm{CCS}_{\sigma}$ processes: $\phi_{S}(P)$ contains only the choices of $P$ that are selected by the scheduler $S$. We now show that the result is indeed a refinement:

Proposition 2. For all $C C S_{\sigma}$ processes $P$ and schedulers $S: \phi_{S}(P) \sqsubseteq P$

Note that $\sqsubseteq$ is the simulation relation on $\mathcal{P}$ wrt the classical CCS semantics $\longrightarrow_{c}$. Also, let $\sim$ be the bisimilarity relation on $\mathcal{P}$ wrt $\longrightarrow_{c}$. A nice property of this type of refinement is that it allows one to refine two processes in a consistent way. This enables us to define a refinement-preserving bisimulation.

Definition 3. An equivalence relation $\mathcal{R}$ on $\mathcal{P}$ is an R-bisimulation iff for all $\left(P_{1}, P_{2}\right) \in$ $\mathcal{R}$ and all finite schedulers $S: \varphi_{S}\left(P_{1}\right) \sim \varphi_{S}\left(P_{2}\right)$. We denote by $\sim_{R}$ the largest $R$ bisimulation.

Note that $P_{1} \sim_{R} P_{2}$ implies $P_{1} \sim P_{2}$ (for $S=0$ ). We now show that processes that preserve bisimilarity under this type of refinement are exactly the ones that are demonic-bisimilar.

Theorem 1. The equivalence relations $\sim_{R}$ and $\sim_{D}$ coincide.

\section{Verifying Demonic Bisimilarity for Finite Processes}

The two characterizations of demonic bisimilarity, given in the previous section, have the drawback of quantifying over all schedulers. This makes the verification of the equivalence difficult, even for finite state processes. To overcome this difficulty, we give a third characterization, this one based on traditional bisimilarity on a modified transition system where labels annotate the performed actions. We then use this characterization to adapt an algorithm for verifying probabilistic bisimilarity to our settings.

\subsection{Characterization Using a Modified Transition System}

In this section we give a modified semantics for $\mathrm{CCS}_{\sigma}$ processes without schedulers. The semantics are given by means of a simple probabilistic automaton with state space 
$\mathcal{P}$, displayed in Figure 4 and denoted by $\longrightarrow_{a}$. The difference is that now the labels annotate the actions instead of being used by the scheduler. Thus, we have actions of the form $\lambda: \alpha$ where $\lambda$ is $l$ or $\left(l_{1}, l_{2}\right)$, and $\alpha$ is a channel, an output on a channel or $\tau$. Note that, in the case of synchronization (COM), we combine the labels $l_{1}, l_{2}$ of the actions $a, \bar{a}$ and we annotate the resulting $\tau$ action by $\left(l_{1}, l_{2}\right)$. All rules match the corresponding transitions for complete processes. Since no schedulers are involved here, the rules IF1 and IF2 are completely removed.

We can now characterize demonic bisimilarity using this transition system.

Definition 4. An equivalence relation $\mathcal{R}$ on $\mathcal{P}$ is an A-bisimulation iff

i) it is a bisimulation wrt $\longrightarrow$, and

ii) $t l\left(P_{1}\right)=t l\left(P_{2}\right)$ for all non-blocked $P_{1}, P_{2} \in \mathcal{R}$

We define $\sim_{A}$ as the largest A-bisimulation on $\mathcal{P}$.

Theorem 2. The equivalence relations $\sim_{D}$ and $\sim_{A}$ coincide.

Essentially, we have encoded the schedulers in the actions of the transition system $\longrightarrow a$. Thus, if two processes perform the same action in $\longrightarrow a$ it means that they perform the same action with the same scheduler in $\longrightarrow_{s}$. Note that the relation $\sim_{A}$ is stricter that the classical bisimilarity. This is needed because schedulers have the power to check the top-level labels of a process, even if this label is not "active", that is it does not correspond to a transition. We could modify the semantics of the if-then-else operator, in order to use the traditional bisimilarity in the above theorem. However, this would make the schedulers less expressive. Indeed, it can be shown ([7]) that for any semantic scheduler (that is, one defined on the automaton) of a $\mathrm{CCS}_{p}$ process $P$, we can create a syntactic scheduler that has the same behaviour on $P$ labeled with a linear labeling. This property, though, is lost under the modified if-then-else.

\subsection{An Algorithm for Finite State Processes}

We can now use $\sim_{A}$ to verify demonic bisimilarity for finite state processes. For this, we adapt the algorithm of Baier ([11]) for probabilistic bisimilarity. The adaptation is straightforward and has been omitted due to space constraints. The only interesting part is to take into account the additional requirement of $\sim_{A}$ that related non-blocked processes should have the same set of top-level labels. This can be done in a pre-processing step where we partition the states based on their top-level labels. More details can be found in the report version of this paper ([9]). The algorithm has been implemented and used to verify some of the results of the following section.

\section{An Application to Security}

In this section, we apply the demonic bisimulation to the verification of anonymity protocols. First, we formalize anonymity in terms of equivalence between different instances of the protocol. We then show that this definition implies strong probabilistic anonymity, which was defined in [12] in terms of traces. This allows us to perform an easier analysis of protocols by exploiting the algebraic properties of an equivalence. We perform such a compositional analysis on the Dining Cryptographers protocol with non-deterministic order of announcements. 


\subsection{Probabilistic Anonymity}

Consider a protocol in which a set $\mathcal{A}$ of anonymous events can occur. An event $a_{i} \in \mathcal{A}$ could mean, for example, that user $i$ performed an action of interest. In each execution, the protocol produces an observable event $o \in \mathcal{O}$. The goal of the attacker is to deduce $a_{i}$ from $o$. Strong anonymity was defined in [12], here we use this definition in a somewhat informal way, a more formal treatment is available in the report version of the paper $([\overline{9}])$.

Definition 5 (strong anonymity). A protocol is strongly anonymous iff for all schedulers, for all $a_{i}, a_{j} \in \mathcal{A}$ and all $o \in \mathcal{O}$, the probability of producing $o$ when $a_{i}$ is selected is equal to the probability of producing $o$ when $a_{j}$ is selected.

Let $\operatorname{Prot}_{i}$ be the $\mathrm{CCS}_{\sigma}$ process modelling the instance of the protocol when $a_{i}$ occurs. Typically, the selection of anonymous event is performed in the beginning of the protocol (for example a user $i$ decides to send a message) and then the protocol proceeds as $\operatorname{Prot}_{i}$. Thus, the complete protocol is modelled by Prot $\triangleq l: \sum_{i} p_{i} \operatorname{Prot}_{i}$. The observable events correspond to the traces of Prot. We can now give a definition of strong anonymity based on demonic bisimulation:

Definition 6 (equivalence based anonymity). A protocol satisfies anonymity iff for all anonymous events $a_{i}, a_{j} \in \mathcal{A}:$ Prot $_{i} \sim_{D}$ Prot $_{j}$.

The idea behind this definition is that, if $\operatorname{Prot}_{i}, \operatorname{Prot}_{j}$ are demonic-bisimilar, they should behave in the same way under all schedulers, thus producing the same observation. Indeed, we can show that the above definition implies Def.5.

Proposition 3. If Prot $\sim_{D}$ Prot $_{j}$ for all $i, j$ then the protocol satisfies strong probabilistic anonymity (Def. 5)

It is worth noting that, on the other hand, $\operatorname{Prot}_{i} \sim \operatorname{Prot}_{j}$ does not imply Def. 5, as we see in the next section.

\subsection{Analysis of the Dining Cryptographers Protocol}

The problem of the Dining Cryptographers is the following: Three cryptographers dine together. After the dinner, the bill has to be paid by either one of them or by another agent called the master. The master decides who will pay and then informs each of them separately whether he has to pay or not. The cryptographers would like to find out whether the payer is the master or one of them. However, in the latter case, they wish to keep the payer anonymous.

The Dining Cryptographers Protocol (DCP) solves the above problem as follows: each cryptographer tosses a fair coin which is visible to himself and his neighbour to the right. Each cryptographer checks the two adjacent coins and, if he is not paying, announces agree if they are the same and disagree otherwise. However, the paying cryptographer says the opposite. It can be proved that the master is paying if and only if the number of disagrees is even ([13]). 


$$
\begin{aligned}
\operatorname{Crypt}_{i} & \triangleq{ }^{1, i} c_{i}\left(\operatorname{coin}_{1}\right){ }^{2, i} c_{i}\left(\operatorname{coin}_{2}\right) .{ }^{3, i} \overline{\text { out }}_{i}\left\langle\operatorname{coin}_{1} \otimes \operatorname{coin}_{2}\right\rangle \\
\text { Crypt }_{i} & \triangleq{ }^{1, i} c_{i}\left(\operatorname{coin}_{1}\right) .^{2, i} c_{i},\left(\operatorname{coin}_{2}\right) .{ }^{3, i} \overline{\text { out }}_{i}\left\langle\operatorname{coin}_{1} \otimes \operatorname{coin}_{2} \otimes 1\right\rangle \\
\text { Coin }_{i} & \triangleq l_{4, i}:\left(\left(\left.{ }^{5, i} \bar{c}_{i}\langle 0\rangle\right|^{6, i} \bar{c}_{i \oplus 1}\langle 0\rangle\right)+0.5\left(\left.{ }^{5, i} \bar{c}_{i}\langle 1\rangle\right|^{6, i} \bar{c}_{i \oplus 1}\langle 1\rangle\right)\right) \\
\operatorname{Prot}_{i} & \triangleq(\nu c)\left(\operatorname{CryptP}_{i} \mid \prod_{j \neq i} \text { Crypt }_{j} \mid \prod_{j=0}^{n-1} \operatorname{Coin}_{j}\right)
\end{aligned}
$$

Fig. 5. Encoding of the dining cryptographers protocol

We model the protocol, for the general case of a ring of $n$ cryptographers, as shown in Figure 5. The symbols $\oplus, \otimes$ represent the addition modulo $n$ and modulo 2 (xor) respectively. Crypt $_{i}$, Crypt $_{i}$ model the cryptographer $i$ acting as non-payer or payer respectively. $\operatorname{Coin}_{i}$ models the $i$-th coin, shared between cryptographers $i$ and $i \oplus 1$. Finally, $\operatorname{Prot}_{i}$ is the instance of the protocol when cryptographer $i$ is the payer, and consists of $\operatorname{Crypt} P_{i}$, all other cryptographers as non-payers, and all coins. An external observer can only see the announcements $\overline{o u t}_{i}\langle\cdot\rangle$. As discussed in [12], DCP satisfies anonymity if we abstract from their order. If their order is observable, on the contrary, a scheduler can reveal the identity of the payer to the observer by forcing the payer to make his announcement first, or by selecting the order based on the value of the coins.

In $\mathrm{CCS}_{\sigma}$ we can be precise about the information that is revealed to the scheduler. In the encoding of Fig. 5, we have used the same labels on both sides of the probabilistic choice in $\operatorname{Coin}_{i}$. As a consequence, after performing the choice, the scheduler cannot use an if-then-else to find out which was the outcome, so his decision will be independent of the coin's value. Similarly, the use of private value passing (see Section 3.3. guarantees that the scheduler will not see which value is transmitted by the coin to the cryptographers. Then we can show that for any number of cryptographers:

$$
\operatorname{Prot}_{i} \sim_{D} \operatorname{Prot}_{j} \quad \forall 1 \leq i, j \leq n
$$

For a fixed number of cryptographers, (11) can be verified automatically using the algorithm of Section (5.2). We have used a prototype implementation to verify demonic bisimilarity for a very small number of cryptographers (after that, the state space becomes too big). However, using the algebraic properties of $\sim_{D}$ we can perform a compositional analysis and prove (11) for any number of cryptographers. This approach is described in the report version of the paper.

This protocol offers a good example of the difference between classical and demonic bisimulation. Wrt the $\longrightarrow_{s}$ transition system, Prot $_{i}$, Prot $_{j}$ are both bisimilar and demonic-bisimilar, and strong anonymity holds. Now let $\operatorname{Coin}_{i}^{\prime}$ be the same as $\operatorname{Coin}_{i}$ but with different labels on the left-hand and right-hand side, meaning that now a scheduler can depend its behaviour on the value of the coin. The resulting $\operatorname{Prot}_{i}^{\prime}, \operatorname{Prot}_{j}^{\prime}$ processes are no longer demonic-bisimilar and strong-anonymity is violated. However, classic bisimulation still holds, showing that it fails to capture the desired security property. 


\section{Related Work}

Various works in the area of probabilistic automata introduce restrictions to the scheduler to avoid violating security properties ([14|15[16]). Their approach is based on dividing the actions of each component of the system in equivalence classes (tasks). The order of execution of different tasks is decided in advance by a so-called task scheduler. The remaining nondeterminism within a task is resolved by a second demonic schedule. In our approach, the order of execution is still decided non-deterministically by a demonic scheduler, but we impose that the scheduler will make the same decision in both processes.

Refinement operators that preserve various security properties are given in [17|18]. In our approach, we impose that the refinement operator should preserve bisimilarity, obtaining a stronger equivalence.

In the probabilistic setting, a bisimulation that quantifies over all schedulers is used in [19]. In this work, however, the scheduler only selects the action and the remaining nondeterminism is resolved probabilistically (using a uniform distribution). This avoids the problem of angelic non-determinism but weakens the power of the scheduler.

On the other hand, [20] gives an equivalence-based definition of anonymity for the Dining Cryptographers, but in a possibilistic setting. In this case the scheduler is clearly angelic, since anonymity relies on a non-deterministic selection of the coins. Our definition is the probabilistic counterpart of this work, which was problematic due to the angelic use of non-determinism.

\section{Conclusion and Future Work}

We introduced a notion of bisimulation where processes are required to simulate each other under the same scheduler. We characterized this equivalence in three different ways: using syntactic schedulers, using a refinement operator based on schedulers and using a modified transition system where labels annotate the actions. We applied this notion to anonymity showing that strong anonymity can be defined in terms of equivalence, leading to a compositional analysis of the dining cryptographers with nondeterministic order of announcements.

As future work, we want to investigate the effect of angelic non-determinism to other process equivalences. Many of them are defined based on the general schema: when $P$ does an action of interest (passes a test, produces a barb, etc) then $Q$ should be able to match it, employing an existential quantifier. Moreover, we would like to investigate models in which both angelic and demonic non-determinism are present. One approach would be to use two separate schedulers, one acting in favour and one against the process, along the lines of [21].

\section{References}

1. Roscoe, A.W.: Modelling and verifying key-exchange protocols using CSP and FDR. In: Proc. CSFW, pp. 98-107. IEEE Computer Soc. Press, Los Alamitos (1995)

2. Abadi, M., Gordon, A.D.: A calculus for cryptographic protocols: The spi calculus. Information and Computation 148, 1-70 (1999) 
3. Abadi, M., Fournet, C.: Mobile values, new names, and secure communication. In: Proceedings of POPL 2001, pp. 104-115. ACM, New York (2001)

4. Kremer, S., Ryan, M.D.: Analysis of an electronic voting protocol in the applied pi calculus. In: Sagiv, M. (ed.) ESOP 2005. LNCS, vol. 3444, pp. 186-200. Springer, Heidelberg (2005)

5. McLean: A general theory of composition for a class of "possibilistic" properties. IEEETSE: IEEE Transactions on Software Engineering 22 (1996)

6. Roscoe, B.: CSP and determinism in security modelling. In: Proc. of 1995 IEEE Symposium on Security and Privacy. IEEE Computer Society Press, Los Alamitos (1995)

7. Chatzikokolakis, K., Palamidessi, C.: Making random choices invisible to the scheduler. In: Caires, L., Vasconcelos, V.T. (eds.) CONCUR 2007. LNCS, vol. 4703, pp. 42-58. Springer, Heidelberg (2007)

8. Segala, R.: Modeling and Verification of Randomized Distributed Real-Time Systems. PhD thesis, MIT (1995)

9. Chatzikokolakis, K., Norman, G., Parker, D.: Bisimulation for demonic schedulers. Technical report (2009), http: / /www.win.tue.nl/ kostas /

10. Chatzikokolakis, K.: Probabilistic and Information-Theoretic Approaches to Anonymity. $\mathrm{PhD}$ thesis, Ecole Polytechnique, Paris (2007)

11. Baier, C.: Polynomial-time algorithms for testing probabilistic bisimulation and simulation. In: Alur, R., Henzinger, T.A. (eds.) CAV 1996. LNCS, vol. 1102, pp. 50-61. Springer, Heidelberg (1996)

12. Bhargava, M., Palamidessi, C.: Probabilistic anonymity. In: Abadi, M., de Alfaro, L. (eds.) CONCUR 2005. LNCS, vol. 3653, pp. 171-185. Springer, Heidelberg (2005)

13. Chaum, D.: The dining cryptographers problem: Unconditional sender and recipient untraceability. Journal of Cryptology 1, 65-75 (1988)

14. Canetti, R., Cheung, L., Kaynar, D., Liskov, M., Lynch, N., Pereira, O., Segala, R.: Taskstructured probabilistic i/o automata. In: Proceedings the 8th International Workshop on Discrete Event Systems (WODES 2006), Ann Arbor, Michigan (2006)

15. Canetti, R., Cheung, L., Kaynar, D.K., Liskov, M., Lynch, N.A., Pereira, O., Segala, R.: Time-bounded task-PIOAs: A framework for analyzing security protocols. In: Dolev, S. (ed.) DISC 2006. LNCS, vol. 4167, pp. 238-253. Springer, Heidelberg (2006)

16. Garcia, F.D., van Rossum, P., Sokolova, A.: Probabilistic anonymity and admissible schedulers, arXiv:0706.1019v1 (2007)

17. Jürjens, J.: Secrecy-preserving refinement. In: Oliveira, J.N., Zave, P. (eds.) FME 2001. LNCS, vol. 2021, p. 135. Springer, Heidelberg (2001)

18. Mantel, H.: Possibilistic definitions of security - an assembly kit. In: CSFW, pp. 185-199 (2000)

19. Lincoln, P., Mitchell, J., Mitchell, M., Scedrov, A.: A probabilistic poly-time framework for protocol analysis. In: Proceedings of the 5th ACM Conference on Computer and Communications Security, pp. 112-121. ACM Press, New York (1998)

20. Schneider, S., Sidiropoulos, A.: CSP and anonymity. In: Martella, G., Kurth, H., Montolivo, E., Bertino, E. (eds.) ESORICS 1996. LNCS, vol. 1146, pp. 198-218. Springer, Heidelberg (1996)

21. Chatzikokolakis, K., Knight, S., Panangaden, P.: Epistemic strategies and games on concurrent processes. In: Geffert, V., Karhumäki, J., Bertoni, A., Preneel, B., Návrat, P., Bieliková, M. (eds.) SOFSEM 2008. LNCS, vol. 4910. Springer, Heidelberg (2008) 